\title{
Philosophiques
}

\section{Les nouvelles philosophies de la nature}

\section{Dominique Lecourt}

Volume 20, numéro 1, printemps 1993

URI : https://id.erudit.org/iderudit/027208ar

DOI : https://doi.org/10.7202/027208ar

Aller au sommaire du numéro

Éditeur(s)

Société de philosophie du Québec

ISSN

0316-2923 (imprimé)

1492-1391 (numérique)

Découvrir la revue

Citer cet article

Lecourt, D. (1993). Les nouvelles philosophies de la nature. Philosophiques, 20(1), 159-176. https://doi.org/10.7202/027208ar d'utilisation que vous pouvez consulter en ligne.

https://apropos.erudit.org/fr/usagers/politique-dutilisation/ 


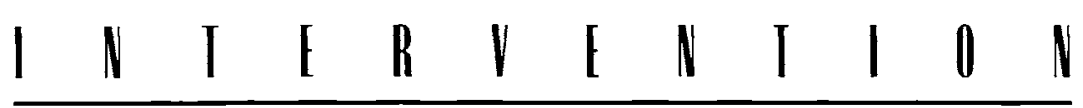

\section{LES NOUVELLES PHILOSOPHIES DE LA NATURE}

\section{par \\ Dominique Lecourt}

En 1956-1957, Maurice Merleau-Ponty consacre son cours du Collège de France au « Concept de nature », il le prolonge l'année suivante et l'achève en 1959-1960, sa dernière année d'enseignement. Il entend réagir contre « l'abandon où est tombée la philosophie de la Nature », abandon imputable à l'hégémonie des philosophies de l'histoire ; lesquelles, suggère-t-il, reposent toutes, comme la plus célèbre d'entre elles, « sur un concept qui n'a jamais été élucidé et qui est peut-être mythique ». Résultat : la nature constitue pour elles une véritable « hantise ».

Trente-cinq ans plus tard, on ne peut qu'admirer le flair du philosophe : le retrait du marxisme et des philosophies de l'histoire se traduit par un grand affairement autour de l'idée d'une philosophie de la nature. J'entends par « philosophie de la Nature » ce que Friedrich Wilhelm Schelling (1775-1854) désigne par cette expression dans ses Idées pour une philosophie de la Nature publiées en 1797 : une science qui abolisse en son sein la distinction instituée par les philosophies de la représentation entre sujet et objet, qui annule la « séparation » entre l'homme et le monde pour accéder à la pure et absolue «productivité » de la Nature.

Si donc nous considérons enfin la Nature comme un Tout, elle se présente à nous sous deux aspects opposés : celui d'un mécanisme, c'est-â-dire d'une série régressive de causes et d'effets, et celui de la 
linalité. c'est-à-dire de l'indépendance de tout mécanisme, d'une simultanéité de causes et d'effets. En réunissant ces deux extrèmes, nous obtenons l'idée d'une finalite du Tout, la Nature devient une ligne circulaire, revenant sur elle-mème, un système clos. La série des causes et effets se trouve interrompue, et l'on obtient a sa place une réciprocité de moyens et de fins. Les parties ne peuvent se. réaliser sans le Tout, ni le Tout sans les parties ISchelling. Idées pour une philosophie de la nature. p. 85].

En relisant de tels textes, on ne peut manquer d'être frappé par la résurgence de leurs motifs et de leurs thèmes essentiels au moins depuis l'ouvrage d'Ilya Prigogine et Isabelle Stengers intitulé La Nonvelle Alliance : Métamorphose de la science (1979). Le motif essentiel : « reconcilier »l'homme avec la nature ; le thème majeur : l'union retrouvée de la science et de la philosophie qui ouvrirait la voie à un réenchantement du monde.

La Nowvelle Alliance propose sur cette base ce qu'il est convenu d'appeler, dans un vocabulaire emprunté à Thomas Kuhn, un « nouveau paradigme », c'est-à-dire un nouveau modèle pour la pensée scientifique ; lequel ferait référence à une « révolution scientifique » accomplie dans les travaux de Prigogine sur les structures dissipatives. D'autres n'hésiteront pas à parler d'une « nouvelle science».

Le retour à la philosophie de la Nature se trouverait donc commandé par un progrès décisif dans les sciences physiques. Il marquerait l'issue de la longue crise qui a affecté depuis près d'un siècle et demi la pensée scientifique aux prises avec le réel.

Parcourons les étapes majeures de cette « crise » afin de porter une appréciation sur cette prétention. On connaît l'accusée : la « science newtonienne », infestée d'un « mécanisme » intempérant, lequel aurait survécu aux grandes secousses dont elle se trouva affectée au début de ce siècle par la constitution de la théorie de la relativité restreinte, puis, plus gravement encore, par la découverte des quanta et l'énoncé en ig27 du (mal) dit «principe d'incertitude » par Heisenberg. On se réfère volontiers pour la caractériser au fameux texte que Pierre-Simon Laplace (1749-1827) a inscrit en tète de son Essai philosophique sur les probabilités rédigé et plusieurs fois remanié par l'auteur entre 1772 et 1825 . La référence est devenue à ce point rituelle qu'on en a comme oublié le contenu mème d'un écrit où l'on découvre la première formulation de ce qu'on appellera plus tard, avec Claude 
Bernard, le « determinisme ». Or ce texte, traité introductif au Calcul des probabilités, se présente comme un véritable manifeste qui vise à faire apparaître la première théorie analytique des probabilités tout à la fois comme la confirmation de l'idéal de la science forgé par la philosophie française des Lumières et comme l'achèvement de l'image de la nature qu'elle avait composée. Laplace s'appuie sur l'éclatant succès qu'il a obtenu grâce au calcul des probabilités pour régler le lancinant problème des perturbations planétaires ${ }^{\mathrm{I}}$. Newton y voyait la main de Dieu. Laplace, lui, n'a plus besoin de cette hypothèse. Il explique par la mécanique seule comment l'ordre de la machine céleste se rétablit de luimême sans qu'on ait recours à quelque intervention providentielle. D'où l'idéal de la science qui s'exprime sous la forme d'un programme : « appliquer les principes et les résultats généraux de la théorie des probabilités aux questions les plus importantes de la vie, qui ne sont en effet, pour la plupart, que des problèmes de probabilité »: ceci vaut pour les « sciences morales » (Laplace, Essai philosophique sur les probabilités, p. II ) où « les causes sont inconnues ou trop compliquées pour que leurs effets puissent être soumis au calcul » : probabilité des témoignages; des choix et des décisions des assemblées ; des jugements des tribunaux; de la mortalité et des durées moyennes de la vie, des mariages et des associations quelconques ; etc. Cet idéal de la science repose sur une capacité indéfinie de prévision grâce à un calcul adéquat effectué à partir de régularités constatées.

A cet idéal se trouve associée une image de la nature intégralement mécanique, dont les lois peuvent être exprimées en

I. Pierre-Simon Laplace dirige ses efforts vers l'analyse des perturbations et de la stabilitè du système solaire. Il démontre en 1787 que la Lune accélère un peu plus sur son orbite qu'on ne l'a expliqué antérieurement ; il attribue cet effet à la diminution de l'excentricité de l'orbite de la Terre, sous l'influence gravitationnelle des autres planètes. Avec le mathématicien Joseph-Louis de Lagrange (1736-1813), il démontre que l'excentricité totale des orbites planétaires du système solaire doit demeurer constante ; si une planète voit son excentricité augmenter, une autre la verra diminuer. Il en va de mêrne pour l'inclinaison des plans orbitaux. Comme la marge des différences entre les excentricités et les inclinaisons est très faible, peu de changements sont possibles. Ceci implique que le système solaire n'a pas été perturbè depuis sa formation et que sa stabilité reste assurée pour des dizaines de milliards d'années à venir, à moins de l'arrivée d'un corps massif. 
termes mathématiques même dans le cas où nous ignorons provisoirement les «véritables causes » qui relient tel événement au « système entier de l'Univers », à cet « ordre de la nature » que célèbre Laplace.

Peut-on identifier la « science newtonienne »à l'image qui en est ainsi proposée, comme on l'a si souvent fait depuis lors à la faveur de l'usage du mot « déterminisme »? La question se pose lorsque l'on se reporte à l'œuvre de Newton lui-même telle qu'elle se présentait un siècle auparavant; elle se pose également lorsque l'on considère la pratique effective de ceux qui ont fait progresser la science dans le sillage de Newton.

L'idéal de la science tel que Newton le formule apparaît sous un tout autre jour : baconien dissident, Newton cherche dans le «livre de la Nature » le dessein de Dieu. Sa « philosophie naturelle » repose certes sur des « principes mathématiques », mais elle a pour horizon une « théologie naturelle » qui exalte la puissance et la sagesse d'un Dieu personnel célèbré comme « seigneur de l'Univers ». L'image de la nature acceptée par Newton s'en trouve profondément marquée. La philosophie naturelle s'incline devant la puissance divine lorsqu'il s'agit de rendre compte de la nature de la force d'attraction ; laquelle n'est pas, à ses yeux, une force physique. Newton, pas plus que Descartes, ne saurait admettre l'idée d'une action immédiate à distance. La nature de cette force ne saurait donc être que « surnaturelle », transphysique disait Koyré. Elle exprime la puissance de Dieu qui établit les relations d'interaction entre les êtres créés dans l'espace vide et absolu qui constitue son «sensorium», c'est-à-dire l'organe général de sa perception.

En chassant Dieu de la mécanique céleste, Laplace a, quant à lui, parfaitement conscience de tourner sur ce point philosophiquement le dos à Newton. De l'explication qu'il apporte à ce qui apparaissait comme des « irrégularités » dans le mouvement des planètes, il tire en effet la conclusion que Dieu est une hypothèse inutile pour expliquer la mécanique céleste. Mais il contribue ainsi à enterrer le mystère de l'« action immédiate à distance » que seuls les efforts d'Ernst Mach puis d'Einstein ramèneront à la conscience du monde savant un siècle plus tard. Plus grave : cet escamotage se fait au nom du progrès des Lumières : Laplace aurait en somme achevé de libérer la science de la tutelle théolo- 
gique et purgé le système newtonien du monde d'une «scorie » liée à ses préjugés religieux. L'opinion, il faut le dire, avait été bien préparée par celui qui fut le véritable introducteur de Newton en France : Voltaire.

L'idéal laplacien de la science se trouve de surcroît biaisé par le type de science qui occupa ce savant mathématicien et astronome. De ses travaux, il ne peut tirer aucune reflexion sur l'expérimentation qui anime la recherche dans les sciences physiques. Observation et calcul apparaissent comme les deux seules procédures qu'il installe au cœur de sa conception de la science. Comme l'a si bien dit Gaston Bachelard : « le déterminisme est descendu du ciel sur la Terre ». Il a de ce fait contribué à donner du travail scientifique une image mystifiée et a sanctifié le tableau de la nature qui lui était associé.

Le malheur aura voulu que cet idéal laplacien, ainsi constitué, ait été adopté par le monde savant presque unanime, comme s'il exprimait la réalité même de l'activité scientifique, et comme si le tableau de la nature qu'il donne était intangible. On note déjà cette sacralisation dans le Cours de philosophie positive (de I830 à I842) d'Auguste Comte (1798-1857). malgré les réserves du polytechnicien sur le calcul des probabilités. Mais le triomphe viendra avec Claude Bernard qui, se référant explicitement à Laplace, écrit dans la célèbre Introduction à létude de la médecine expérimentale (1865) : « Dans les corps vivants comme dans les corps bruts, les lois sont immuables, et les phénomènes que ces lois régissent sont liés à leur condition d'existence par un déterminisme nécessaire et absolu ».

Claude Bernard avait conscience d'avoir effectué une prouesse philosophique en construisant le concept scientifique de «milieu intérieur ». Pour prendre la mesure de cette prouesse, et de ses suites, il convient de revenir, en deça du texte de Laplace, à ce que fut l'immense impact de l'ceuvre de Newton dans les sciences du vivant, et de ne point oublier l'ambition qui fut celle des naturalistes pendant un siècle et demi de devenir les « Newton de l'histoire naturelle». Mais cette ambition avait cherché à se réaliser selon deux voies divergentes selon qu'on était en Angleterre ou sur le continent. L'Angleterre reste le royaume de la «Théologie naturelle », et les naturalistes, qui y sont en majorité des pasteurs, y annexent leur discipline. $\mathrm{L}^{\prime} \ll$ organi- 
sation $\gg$ du vivant manifeste par excellence le dessein du Créateur. Un maitre mot s'y impose : celui d'« adaptation ». Mar que de la perfection interne de l'organisme dont l'ajustement des parties entre elles et des parties au Tout n'a d'égale que celle dé l'organisme à son milieu. Ce thème lancé par William Derham dans sa retentissante Physico-Theology publiée en i7i3 se trouve exalté par l'archidiacre William Paley dans sa Natural Theology ( 1802$)$, le livre où s'instruiront tous les naturalistes anglais du début du XIX' siècle, Darwin compris.

En France, même lorsque l'on est anglophile en politique, on récuse ce type de finalisme; le déisme ne fait pas bon ménage avec le théisme. Ni Buffon ni Cuvier, par exemple, ni Diderot ne se rallient à une telle démarche. S'ils se veulent les uns et les autres newtoniens, leur projet consiste, sous des formes diverses, à saisir le vivant comme un système de forces reliant des points matériels qui puissent être exprimées en termes mathématiques. Or, ce chemin semblait avoir été définitivement barré, au moment même oú Laplace rédigeait son texte, par un philosophe dont le nom se trouvait en cosmologie associé au sien : Emmanuel Kant. L'auteur d'abord anonyme de l'Histoire générale de la nature et théorie du ciel ( 1755 ), où se trouvait formulee la première hypothèse nébulaire de la formation du système solaire sur la base d'une critique du finalisme newtonien, avait ferraillé pendant près de quarante ans contre tout projet de « théologie naturelle » jusqu'à en faire apparaître le projet comme un « délire » de la raison pure, une «illusion transcendantale » reposant sur un mauvais usage des facultés de connaître. Il en était venu dans la Critique de la faculté de juger (1789) à frapper d'interdit toute extension pure et simple du «mécanisme » au vivant. La question, à vrai dire, n'a rien de simple lorsqu' on veut en suivre la formulation dans les textes que lui consacre Kant, ainsi que Gérard Lebrun en a fait la démonstration magistrale. N'en retenons qu'un aspect. Dans cet ouvrage, Kant qui conserve la tripartition des règnes « animal-végétalminéral » et qui réserve le qualificatif du « vivant » au premier, n'en identifie pas moins un caractère commun aux êtres organisés (animaux et végétaux) : l'auto-organisation. Ce qui lui permet de les désigner comme « fins naturelles »: « un être organisé et s'organisant lui-même pourra être nommé fin naturelle ». Mais qui dit « fin », dit « art ». Comment penser les organismes comme 
les produits d'un art, mais non comme des produits artificiels? Autrement dit : quel concept forger d'un causalité organique qui se présente comme une technique, sans ètre une fabrication? ou encore : quel sens faut-il donner au mot vie pour respecter le fait de sa finalité interne sans le réduire à une finalité intentionnelle? Si Kant n'apporte pas la réponse, il récuse tout modèle mécanique du vivant. Les $\S \S 64$ et 65 de la Critique de la faculté de juger sont sur ce point les textes clés. Il n'y aura pas de « Newton de brin d'herbe », mème si, dégagé par l'idée régulatrice d'une finalité naturelle, un espace s'ouvre où viendra positivement se loger la future biologie.

La déclaration de Claude Bernard sonne ainsi comme la levée triomphale d'un interdit et l'accomplissement d'un vœu. Le «déterminisme nécessaire et absolu » qu'il proclame s'affiche comme la double déroute du « fatalisme », c'est-à-dire du réductionnisme physico-chimique, et du vitalisme qui croyait à l'existence d'une mystérieuse « force vitale » animant la matière vivante.

Mais au moment où le succès de la conception laplacienne de la science semblait ainsi assuré, on le voyait subir son premier coup, avec la redécouverte, gràce à Clapeyron, du mémoire de Sadi Carnot intitulé « Réflexions sur la puissance motrice du feu et sur les machines propres à développer cette puissance » (I824). Un aspect essentiel de la mécanique rationnelle « newtonienne » se trouve brutalement remis en question. Avec les phénomènes de transformation de la chaleur en mouvement, on découvre dans la nature l'existence de processus irréversibles. Il apparait dès lors que la vision newtonienne reposait sur un postulat tacite : celui de la réversibilité de tous les mouvements. Ainsi s'ouvre ce que de nombreux physiciens parmi les plus éminents du temps ont appelé « la crise de la physique moderne ». Elle dura à vrai dire près de cinquante ans!

Il n'est pas sans intèrèt de tirer aujourd'hui de l'oubli où elles sont tombées les argumentations de ces savants en détresse. Sur la base d'une analyse critique des présupposés philosophiques de ce qu'ils appelèrent dès lors le « mécanisme » de la physique antérieure, ils en vinrent en effet à proposer une grandiose «philosophie de la nature » sous le nom d'énergétisme. Le grand chimiste Wilhelm Ostwald en fut l'artisan majeur, prolifique et 
militant. Dans ses Vorlesungen iiber Naturphilosophie parues à Leipzig en rgoz et traduites en français sous le titre (révélateur dans sa trahison) de : Esquisse d'ume philosophie des sciences en 19I I, Ostwald tire la leçon du second principe. Si nous avons admis le postulat de la réversibilité des mouvements, c'est que nous avons adhéré à la conception newtonienne de l'espace et du temps absolus qui pourtant restaient aux dires mèmes de Newton un «mystère insondable » au coeur de sa philosophie naturelle. Et c'est ainsi que nous avons logé les « choses » dans cet espace et dans ce temps en les considérant comme les supports passifs et indéterminès de propriétés observables; la conception de la matière que nous en avons tirée n'était ainsi qu'une pure abstraction où nous avons trouvé à chosifier nos impressions sensibles en leur accolant des « marques » conceptuelles purement symboliques.

La thermodynamique nous découvre une autre réalité que nous pouvons substituer à la notion abstraite de matière. Nous tenons là enfin le foyer actif du réel : l'énergie. Ernst Cassirer commente admirablement :

Il n'y a plus désormais aucun symbole pour se glisser entre nous et les choses du monde physique : nous ne sommes plus dans le domaine de la simple pensée. nous hantons celui de l'être. Et nous n'avons nul besoin desormais, pour tenir cet être ultime, de faire détour par des hypothèses mathèmatiques compliquées; il se révèle à nous directement et sans que nous ayons besoin de le traquer dans la perception mème ISubstance et fonction, Berlin, Igıo, trad. franç. 1977. p. 220l.

De frit. Ostwald explique très clairement que

I... la nalure dans sa totalité nous apparaît sous la forme d'énergie spatialement et temporellement variables, réparties selon l'espace et le temps, énergies dont nous ne prenons connaissance que dans la mesure où elles se transmettent à notre corps et tout spécialement aux organes des sens préparés à les recevoir lOstwald, Esquisse d'une philosophie des sciences, igo2, p. I59 sq.l.

Voici l'homme réconcilié avec la nature ! Non seulement toute la physique peut ètre reconstruite à partir du rapport d'équivalence établi entre mouvement et chaleur, mais aussi la chimie, la biologie, la psychologie, la sociologie et l'esthétique. Telle apparaît donc la première grande « philosophie de la nature » élaborée par des scientifiques et dont l'empire sur les esprits fut immense. Elle se construit en réponse à une faille découverte dans l'édifice de la physique. 
A s'en tenir à la part la plus manifeste du rôle qu'elle a tenu dans l'histoire de la physique, on souligne souvent, à juste titre, qu'Ostwald a jeté toutes ses forces dans la bataille contre l'« hypothèse atomique » au plus mauvais moment. Les énergétistes se trouvèrent à Vienne aux côtés des disciples d'Ernst Mach pour combattre la thermodynamique statistique de Boltzmann. La France de Marcelin Berthelot fit bon accueil aux écrits d'Ostwald qui contribuèrent au retard historique pris par la chimie française au début du siècle, pour le plus grand bénéfice de l'industrie allemande. Mais il ne faut pas négliger la fonction critique puissante qu'elle a pu remplir contre la conception substantialiste et dogmatique de la matière qui dominait la pensée des physiciens. Ostwald se flattait de sortir la physique moderne de sa crise prolongée. Mais le véritable dénouement fut l'œuvre d'Einstein par son coup d'audace de I905, au prix d'une refonte autrement plus radicale de la physique newtonienne. Einstein n'a jamais caché sa dette à l'égard de Mach : il trouva dans La mécanique (igo2) une critique du mécanisme de la physique classique qui le mit sur la voie. L'accent y portait sur le mystère des « forces à distance ». On s'y habitua, écrit Mach, et

[...] finalement cette action à distance des particules séparées supposées constituer les corps servit même à expliquer la résistance qu'ils opposent à la pression et au choc, c'est-à-dire à l'action au contact : en fait, à cause de la discontinuité, cette dernière action est représentee comme une fonction plus compliquée que la première. C'est ainsi que chez Laplace et ses contemporains les formes à distance furent universellement en faveur.

S'étant engagé dans un exposé historique et critique du développement de cette science, Mach s'interroge sur la genèse de l'erreur de Newton : il montre comment la notion de masse adoptée par Newton « restait psychologiquement très voisine de celle de quantité de matière ». Après quoi vient le chapitre décisif sur «les idées newtoniennes sur le temps, l'espace et le mouvement ». De Newton, Mach remonte à Galilée et produit en quelques pages un « Newton lecteur de Galilée » que retiendra Finstein dans sa lecture de Newton. L'essentiel me paraît être qu' Einstein n'emprunte pas pour sa part la voie d'une « philosophie de la nature »: pardelà un Newton décapé de ses propres présupposés et de son habillage philosophique kantien, il renoue le fil avec la pensée galiléenne de la relativité pour en étendre le principe dans le cadre 
d'une théorie qui rend compte de sa limitation initiale. Moyennant quoi, il pouvait lever les difficultés qui s'étaient accumulées sur la nature de la lumière depuis que James Clerk Maxwell ( $1831-1879$ ) avait formulé les équations majeures de la théorie de l'électromagnétisme, et libérait la pensée des physiciens des apories que soulevaient des expériences, comme celle de Michelson et Morley, qui mettaient en cause la notion «psychologiquement » évidente de simultanéité. Mais si déconcertante qu'ait pu paraitre la notion d'« espace-temps», elle ne procédait qu'à une refonte de l'image laplacienne de la nature. On le vit bien lorsque Einstein recula devant sa propre audace et entreprit de forger un scénario cosmogonique correspondant à la théorie relativiste de la gravitation. Il ne touchait nullement à l'idéal de la science qui s'était imposé sous le nom de déterminisme.

Ce fut la mécanique quantique qui porta à cet idéal un coup très rude. Non, comme on le dit souvent, parce qu'elle aurait, avec les travaux de Max Planck sur le rayonnement du « corps noir » en 1900, introduit le discontinu dans la nature. Depuis Newton au moins, l'idée d'une discontinuité de la matière, objet de scandale pour Aristote et ses successeurs, était familière au monde savant, même si elle restait de l'ordre d'une hypothèse hautement raisonnable. L'affaire était en réalité beaucoup plus grave : la formation du principe dit d'« incertitude » par Werner Heisenberg en 1927 établissait sur une mathématique imparable qu'il serait à jamais impossible de préciser tout à la fois la position et la vitesse d'un électron ou d'une particule quantique quelconque. L'idée, avancée par Laplace, qu'il suffirait d'«appliquer » les lois de la physique à des domaines toujours nouveaux pour progresser vers une connaissance de plus en plus exacte de la nature se trouvait ainsi mise en échec. L'ambition d'une « prévision » portant sur l'évolution de tout système physique et de l'univers lui-même, essentielle au déterminisme, semblait subir un démenti direct et irrémédiable.

À l'épreuve de la nouvelle crise qui affecte la physique moderne avec la constitution de la mécanique quantique, Einstein se révèle en un sens plus laplacien que Laplace. Que la probabilité règne dans le monde subatomique lui apparaît intolérable. Il retrouve en tout cas les termes mêmes de 
l'astronome français pour imputer cette situation à notre ignorance (provisoire) des causes. Que la Nature se révèle scindée en deux régions soumises à des lois irréductibles les unes aux autres, voilà qui brise l'image unifiée de la nature qui se trouve au cour, sinon au principe, de la théorie de la relativité.

Quoi qu'il en soit du combat d'Einstein, la crise ouverte par la mécanique quantique n'a cessé de s'aggraver de 1900 à 1927. On connaît les signifiants majeurs de ce nouvel état de crise: « indéterminisme » (mot forgé pour l'occasion), « principe d'incertitude ». Or, à relire les pièces du « grand débat », on ne peut manquer d'être frappé d'un étrange paradoxe. À partir de I927, il se concentre sur l'interaction entre le système d'observation et le système observé. Dès lors que cette interaction s'avère mathématiquement inesquivable, le mot d'incertitude apparaît dans la littérature. Ce mot porte en lui-même l'écho d'une déception par rapport à l'idéal laplacien qui s'ordonnait autour de l'idée de certitude. De la fameuse « intelligence » évoquée dans l'Essailaquelle n'est certes pas un « démon », mais plutôt le fantôme de l'hypothèse-Dieu - il était dit qu'« à ses yeux rien ne serait incertain ». Mais, au même moment, la précision de la mécanique quantique s'averait extraordinaire. Thème constant de Victor Weisskopf (La révolution des quanta): la mécanique quantique a introduit dans le monde un principe d'identité inédit. Elle explique par exemple pourquoi un atome d'or est un atome d'or où que ce soit, quelle qu'ait été son histoire.

D'où un double discours : celui de l'incertitude, qui se déploie philosophiquement sous les espèces d'un immaterialisme renouvelé. Si vous identifiez le système subatomique soumis à la mesure à l'« objet » et le système de mesures à l'« observateur », c'est-à-dire au « sujet », voire à la « conscience » ou à « l'esprit » de celui-ci, vous faites apparaître que, par principe, le sujet ne saurait connaître que les effets de ses propres interventions dans le réel qui restera à jamais « voilé » (D’Espagnat); un pas de plus, et vous direz que c'est lui-mème, ses pouvoirs, que l'esprit retrouve en la matière et rien d'autre. Costa de Beauregard n'est pas loin.

Mais parallèle à ce discours, un autre se déploie d'une tonalité contraire qui célèbre la conquête de l'infiniment petit par la puissance des concepts physiques mathématiquement informés. Si l'on récuse toute considération «métaphysique » sur le réel 
comme question mal posée imputable aux mystifications du langage ordinaire, cette célébration peut prétendre s'inscrire dans le droit fil laplacien. Le Cercle de Vienne, fondé sous ce nom en I929, assume cette prétention.

Il n'y a pas que les discours : il y a les impressionnantes, et bientôt terrifiantes, réalisations de cette science prétendue « incertaine ». D'où le fait que nulle philosophie de la nature ne parvient à s'imposer dans l'immédiat, malgré les efforts, par exemple, de ce Stéphane Lupasco que certains redécouvrent aujourd'hui. Mais n' oublions pas le diagnostic de Merleau-Ponty: l'hégémonie des philosophies de l'histoire joue son rôle dans ce blocage.

On ne voit la philosophie de la nature renaître qu'à compter des années soixante. Il me semble que plusieurs éléments permettent d'en rendre compte. Le premier tient à l'histoire de la cosmologie. Ig64 : telle est la date de la découverte accidentelle du rayonnement du fond du ciel. Désormais, la théorie de l'expansion de l'univers, élaborée à partir des observations sur la fuite des galaxies dues à Edwin Powell Hubble (rg2g) et interprétée après coup dans le cadre de la théorie relativiste de la gravitation, paraît assurée. Elle semble mettre un terme aux grandes discussions qui avaient marqué la première moitié de ce siècle, le scénario dit du «Big-Bang » semble s'imposer. Mais du même coup ressurgissent à propos de l'univers les questions d'origine et de fin dont le XvII ${ }^{\cdot}$ siècle avait cru pouvoir débarrasser ses «systèmes du monde ».

Second élément : les sciences du vivant avaient traversé une longue période de déchirement. Darwin avait bien été enterré en I882 à Westminster avec le titre longtemps convoité de « Newton de la biologie », mais la redécouverte des travaux de Mendel par De Vries, Correns et Tschermack dans les premières années du siècle, puis les travaux de Morgan sur la drosophile (rgzo) avaient introduit un trouble considérable : comment concilier l'idée de «mutation » avec une théorie de l'evolution qui s'affichait philosophiquement « continuiste »? Les biologistes se divisèrent durablement en « néo-lamarckinistes » et en « néo-darwinistes » selon une ligne de partage fondée sur un malentendu (la fameuse question des « caractères acquis »). William Bateson en viendra à parler, dans les années trente, d'une « éclipse du 
darwinisme ». Or, on voit triompher au cours des années quarante ce que Julian Huxley appelle « the new synthesis » et qu' on désignera sous le nom de «théorie synthétique de l'évolution ». Ernst Mayr a montré avec lyrisme comment l'union de la génétique et de la biologie moléculaire, dont le projet avait été formulé par Erwin Schrödinger dans son ouvrage Quiest-ce que la vie? (1944), constituait le cour de cette théorie, laquelle triomphe avec les découvertes de Watson et Crick sur la structure de l'ADN et la constitution « génétique des populations ».

Voici la « vie » et son histoire qui semblent s'intégrer à la vision quantique de la nature.

Troisième élément : la notion de «programme », arrachée à son passé presque exclusivement theâtral, s'introduit dans le lan gage scientifique sous la plume du mathématicien anglais Alan Turing, dans son célèbre article de rg36 sur les « nombres calculables » dont les présupposés philosophiques sont d'ailleurs assumés comme «laplaciens». «Que signifie penser pour une machine? » se demande Turing. A cette question bien délimitée fait écho un rève que les fondateurs de l'intelligence artificielle, vingt ans plus tard à Darmouth, croient pouvoir commencer à réaliser : celui d'éclairer les mécanismes de l'intelligence par l'intelligence de la machine. Le pouvoir d'envoùtement de ce rêve s'avère si fort que son motif essentiel semble se trouver renforcé par l'apparition de la première théorie de l'information, et de son développement rapide depuis la publication de l'article de Claude Shannon intitule « Une analyse symbolique des relais et des communications de circuits $»$. Cet article établissait le lien entre les mathématiques binaires, la logique symbolique et le fonctionnement des circuits électroniques. L'idée d'information se généralise comme « progrès d'un ordre structural efficace ». L'entité désignée par Shannon devient accessible à la mesure. Elle apparaît comme le contraire d'une destruction, d'une diminution d'ordre. Mais en physique, depuis Clausius mais surt out Boltzmann, une dirninution d'ordre, cela porte le nom d'« entropie ». La jonction s'opère sans difficulté entre ces recherches technologiques et les recherches théoriques en intelligence artificielle; mais aussi avec la théorie synthetique de l'évolution, à la faveur de la notion de programme, puis de « code » génétique introduite dans un sens encore juridique par Schrödinger. Ernst Mayr écrit pudiquement : 
« la percée de la biologie moléculaire dans les années I940-1950 coïncida avec la naissance de la science de l'information et certains des mots clés de cette discipline passèrent dans la génétique moléculaire $\gg$. Il ne s'agit pas de vocabulaire en cette coïncidence, mais d'un échange intense de concepts à travers la notion de programme ; laquelle importe dans la biologie une part essentielle des visées théoriques au service desquelles cette notion avait été formée : rendre compte du fonctionnement d'une machine finalisée à rétroaction. Ce qui a facilité. en un premier temps, cet échange fut que la physiologie avait trouvé beaucoup à apprendre des travaux des cybernéticiens : le montage des rétroactions avait notamment permis de faire apparaître le montage en feed-back des connexions nerveuses. L'homme « neuronal » se profilait ainsi déjà à l'horizon. Tous les éléments se trouvaient désormais reunis pour la constitution d'une nouvelle «philosophie de la nature »: du scénario d'évolution d'un univers à nouveau fini au fonctionnement programmé de la pensée humaine par le biais d'une vue de l'organisme qui le présente comme tout entier s'autoconstruisant sur la base d'un organigramme génétique, le développement des formes vivantes se trouvant tributaire d'une information contenue dans les gènes de la cellule initiale.

Cet ensemble théorique trouve un premier couronnement philosophique dans le fameux livre de Jacques Monod, Le hasard et la nécessité (1970). Significativement, l'auteur qui emprunte son titre à Démocrite qualifie sa réflexion de « philosophie naturelle », au bénéfice d'une « éthique » austère qui présente l'homme « seul dans l'univers ». Mais neuf ans plus tard, on voit les mèmes éléments se recomposer selon une tout autre perspective. Michel Serres, qui avait célébré Jacques Monod, se joint à Prigogine lorsqu'il découvre dans les travaux de celui-ci le moyen d'arrimer la théorie synthétique de l'évolution à la thermodynamique statistique. Il écrit dans Hermès III, « La traduction »:

La biochimie est une chimie comme une autre, au moins dans ses méthodes et son épistémologie ; la chimie est une science par la physique, au moins depuis Jean Perrin. et la philosophie de la physique. cest la théorie de l'information. Et donc lorsqu'un biochimiste annonce qu'il écrit une philosophie naturelle, cela signifie en clair qu'il applique la théorie de l'information (philosophie naturelle de la “ philosophie naturelle ») à sa discipline propre. 
Prigogine et Stengers ne manquent pas de saluer Serres. Ils ont parfaitement raison. Une « philosophie de la nature » est née qui peut se prévaloir d'annuler la « séparation » de l'homme du monde, comme naguère celle de Schelling.

Philosophiquement, les auteurs de La Nouvelle Alliance se réfèrent avec insistance à Bergson, lequel se trouve être dans la philosophie du XXe siècle le plus notable des disciples du philosophe allemand. Michel Serres inscrit, pour sa part, son œuvre à l'enseigne de Leibniz; c'est à Leibniz contre Kant et contre Fichte, et si je puis dire, tout contre Spinoza, que Schelling donne raison lorsqu'il s'agit de penser l'Absolu.

le système de Spinoza fut la première prospection hardie d'une imagination qui deduisit directement le fini de l'idée de l'infini et reconnut celui-là comme implique dans celui-ci. Leibniz vint et suivit un chemin opposé. Le temps est venu de réhabiliter sa philosophie (Schelling, Idées, p. 53.).

La nouvelle philosophie de la Nature installe à son principe le concept d'« auto-organisation », c'est-à-dire d'un ordre qui se crée lui-même à partir du désordre; figure contemporaine de l' Absolu, ce concept connaît une expansion qui ne saurait être limitée puisqu'il est destiné à penser le Tout, puisqu'il s'affiche comme principe de la connaissance positive du Tout, laquelle s'accomplit lorsque aucun écart ne subsiste plus entre elle et ce Tout dont elle provient sans en sortir.

Les phénomènes dits de turbulence ou de chaos, dont les physiciens ont pu s'emparer à partir des travaux sur ordinateur du météorologue américain Edward Lorenz en rg63, au MIT, et du physicien français David Ruelle, se trouvent ainsi invités à s'inscrire dans cette philosophie. Ces travaux sont nés d'une surprise: l'étude du mouvement convectif d'une couche d'air dans l'atmosphère réputée hors de portée de la physique classique se revélait soudain susceptible d'une formalisation mathématique très simple correspondant à un système à trois degrés de liberté seulement. Mais la surprise s'accentua lorsqu'il apparut que ce système « classique » - au sens où il est non quantique - s'avérait intrinsèquement probabiliste. Elle atteignit son comble lorsqu' on dut constater que de tels systèmes n'avaient rien d'exotique : une multitude d'autres systèmes dont on attendait classiquement un mouvement régulier s'avéraient en réalité susceptibles de présenter ainsi un comportement erratique. On y trouva la confirmation 
du pressentiment qu'avaient eu au siècle dernier les mathématiciens français Jacques Hadamard et Henri Poincaré. La théorie du chaos qui découvre un ordre dans le désordre même paraît une aubaine pour notre philosophie de la nature.

Peut-être devrait-elle engager la pensée sur une tout autre voie; elle pourrait nous inviter à achever de nous libérer des derniers présupposés de l'idéal laplacien de la science et, du même coup, de la « hantise » de la « nature », ce concept mythique qui marque depuis ses débuts l'histoire de la science moderne. Lorsque Laplace identifiait la science à la prévision rationnelle, il supposait que la connaissance des conditions initiales du systeme considéré pouvait être « exacte ». Or l'explication des phénomènes de chaos montre que des différences infimes dans les dites conditions, insaisissables par les mesures classiques, peuvent produire des effets extrêmement différents dans un système donné. Poincaré avait raisonné sur un caillou lâché du sommet d'une colline: un écart minime dans la direction de la poussée initiale pouvait le mener, en fin de course, à des positions distantes de plusieurs kilomètres. On a maintenant étudié de nombreux systèmes en situation dite de « dépendance sensible aux conditions initiales $\gg$. Comme il est possible avec une mathématique adéquate (celle qui traite des fonctions non linéaires) de prévoir leur comportement avec toute la précision souhaitable, on a voulu parler de « chaos déterministe ».

Mieux vaudrait remettre en perspective toute l'histoire de ce qu'on a appelé le « déterminisme ». L'idée que la science dût être, sur le modèle de la mécanique céleste, calcul sur des régularités observées a déjà été mise à mal par la mécanique quantique. Gaston Bachelard le faisait déjà remarquer il y a plus d'un demi-siècle: la physique a toujours construit ses objets; cette construction. qui s'effectue à travers un dispositif expérimental, se traduit toujours par une découpe des phénomènes, laquelle demande qu'on en écarte un certain nombre de traits tenus par décision pour « négligeables ». L'image laplacienne de la mécanique rationnelle s'avère n'avoir jamais été qu'une fiction, chaque jour démentie par le travail effectif des savants. De découpes en découpes, et de recoupements en recoupements, les physiciens ont tracé les lignes d'horizon qui correspondent au point où ils jugent bon c'est-à-dire fécond - de situer leur point de vue. Ce jugement 
représente toujours un risque, le risque d'une pensée qui ne dispose d'aucune certitude quant à la possibilité d'avérer, en lui conférant le caractère de la nécessité, le possible qu'elle suppute: l'argumentation qui soutient cette prise de risque s'appelle en Occident «philosophie ».

Le fantôme de « la Nature » ne figurerait-il pas dans la pensée moderne un mouvement de fuite devant ce risque, devant l'inconfort d'une irréductible incertitude? On comprendrait dans ces conditions les ressorts intimes de toute «philosophie de la Nature »: qu'épistémologiquement la philosophie se mue en science, science spéculative, science absolue (Schelling); que l'univers, considéré comme un Tout, se trouve doté d'une «finalité » interne qui lui assigne un sens, lequel se manifeste au mieux dans l'ordre du vivant, et tout spécialement dans l'être de ce vivant supposé suprême que serait l'homme.

Les «philosophies de la Nature » ont procédé à une critique souvent féconde du mécanisme laplacien et de sa descendance positiviste, mais elles ont conservé de lui l'idée d'un « ordre de la nature » à découvrir. Cet ordre est supposé conjurer a priori la contingence. Lorsque avec Darwin, le hasard apparaît au principe de l'histoire de la vie, on recule d'horreur aussi bien du côté des théologiens naturalistes anglais que du côté des évolutionnistes lamarckiens athées français. Et Marx lorsqu'il songe à enrôler le darwinisme comme « base naturelle » de sa philosophie de l'histoire cherche à l'expurger du hasard des variations. L'énergétisme croit avoir trouvé les moyens de le conjurer. Et la « théorie synthétique de l'évolution » encore à sa façon. Le vocabulaire de la « loi » appliqué à la nature exprimerait avec constance la persistance de ce mouvement de fuite.

Pourtant sans cesse la contingence vient bouleverser cette figure de l'ordre, et sans cesse les chercheurs inventifs se trouvent invités à risquer le tout de leur pensée dans un jugement sans garantie de succès.

Voilà pourquoi, sans doute, il serait temps de reprendre le chemin de la «philosophie naturelle »-expression qui signait à l'aube des temps modernes - la présence de l'argumentation philosophique et de la connaissance scientifique à l'intérieur d'une même pensée. Les philosophies de la Nature apparaittraient alors comme un positivisme inversé qui a toujours été appelé à 
nourrir des flambées d'enthousiasme de caractère confusément religieux, même lorsqu'elles se sont réclamées du matérialisme le plus radical. Nous en avons les résultats inquiétants sous les yeux : le «New Age » fait bon ménage avec la soi-disant « nouvelle science ». Ne s'agit-il pas dans les deux cas de s'en remettre de son destin à quelque Absolu où se fondre?

Professeur des Universités

Paris 